\title{
Ovarian cancer stem cell-specific gene expression profiling and targeted drug prescreening
}

\author{
YUTING HUANG $^{1,2^{*}}$, BAOHUI JU ${ }^{1,3^{*}}$, JING TIAN ${ }^{1}$, FENGHUA LIU ${ }^{1}$, HU YU $^{1}$, HUITING XIAO ${ }^{1}$, \\ XIANGYU LIU ${ }^{1}$, WENXIN LIU ${ }^{1}$, ZHI YAO ${ }^{2}$ and QUAN HAO ${ }^{1}$
}

\begin{abstract}
${ }^{1}$ Department of Gynecological Oncology, Tianjin Medical Univerisity Cancer Institute and Hospital, National Clinical Research Center of Cancer, Key Laboratory of Cancer Prevention and Therapy, Tianjin 300060; ${ }^{2}$ Department of Immunology, Tianjin Key Laboratory of Cellular and Molecular Immunology, Key Laboratory of Educational Ministry of China, School of Basic Medical Sciences, Tianjin Medical University, Tianjin 300070, P.R. China
\end{abstract}

Received November 26, 2013; Accepted December 23, 2013

DOI: 10.3892/or.2014.2976

\begin{abstract}
Cancer stem cells, with unlimited self-renewal potential and other stem cell characteristics, occur in several types of cancer, including ovarian cancer $(\mathrm{OvC})$. Although CSCs can cause tumor initiation, malignant proliferation, relapse and multi-drug resistance, ways to eliminate them remain unknown. In the present study, we compared ovarian cancer stem cell (OVCSC) expression profiles in normal ovarian surface epithelium and ovarian cells from patients with advanced disease to identify key pathways and specific molecular signatures involved in OVC progression and to prescreen candidate small-molecule compounds with antiOVCSC activity. Comparison of genome-wide expression profiles of OvC stemness groups with non-stemness controls revealed 6495, 1347 and 509 differentially expressed genes in SDC, SP1 and SP2 groups, respectively, with a cut-off of fold-change set at $>1.5$ and $\mathrm{P}<0.05$. NAB1 and NPIPL1 were commonly upregulated whereas PROS1, GREB1, KLF9 and MTUS1 were commonly downregulated in all 3 groups. Most differentially expressed genes consistently clustered with
\end{abstract}

Correspondence to: Dr Quan Hao, Department of Gynecological Oncology, Tianjin Medical Univerisity Cancer Institute and Hospital, National Clinical Research Center of Cancer, Key Laboratory of Cancer Prevention and Therapy, West Huanhu Road, Hexi, Tianjin 300060, P.R. China

E-mail: haoquan1967@gmail.com

Dr Zhi Yao, Department of Immunology, Tianjin Key Laboratory of Cellular and Molecular Immunology, Key Laboratory of Educational Ministry of China, School of Basic Medical Sciences, Tianjin Medical University, 22 Qixiangtai Road, Heping, Tianjin 300070, P.R. China E-mail: yaozhi@tijmu.edu.cn

Present address: ${ }^{3}$ Department of Family Planning, The Second Hospital of Tianjin Medical University, Tianjin 300211, P.R. China

\section{${ }^{*}$ Contributed equally}

Key words: ovarian cancer, cancer stem cell, ovarian cancer stem cell, gene expression profile, molecular signature, drug screening molecular functions such as protein receptor binding, kinase activity and chemo-repellent activity. These genes regulate cellular components such as centrosome, plasma membrane receptors, and basal lamina, and may participate in biological processes such as cell cycle regulation, chemoresistance and stemness induction. Key Kyoto Encyclopedia of Genes and Genomes (KEGG) pathways such as ECM receptor, ErbB signaling, endocytosis and adherens junction pathways were enriched. Gene co-expression extrapolation screening by the Connectivity Map revealed several small-molecule compounds (such as SC-560, disulfiram, thapsigargin, esculetin and cinchonine) with potential anti-OVCSC properties targeting OVCSC signature genes. We identified several key CSC features and specific regulation networks in OVCSCs and predicted several small molecules with potential anti-OVCSC pharmacological properties, which may aid the development of OVCSC-specific drugs.

\section{Introduction}

Cancer stem cells (CSCs) have the ability to self-renew and generate heterogeneous lineages of cancer cells in a tumor. In contrast to other cell populations in a tumor, this small fraction of cells is highly tumorigenic. CSCs can survive chemotherapy and radiotherapy via efficient DNA repair and various drug-pumping mechanisms (1). Therefore, elimination of these rapidly replicating cancer cells is important to ensure successful cancer treatment. Failure of primary treatments to kill a sufficient number of CSCs can lead to relapse and metastasis, often with chemoresistance (2). In ovarian cancer (OvC), the third most common gynecological malignancy, primary cytoreductive surgery in combination with chemotherapy is initially effective. However, most patients develop drug resistance and eventually relapse within 18 months of treatment (3). Due to relapse and metastasis and the potential involvement of CSCs, the 5-year survival rate of patients with OvC continues to be $<30 \%$ and their comprehensive mortality is the highest among all gynecological malignant tumors.

Ovarian cancer stem cells (OVCSCs) were first isolated and characterized after the discovery of CSCs in leukemia (4). Since then, techniques such as isolation of side population 
(SP) through flow cytometry and in vitro culture of ovarian multiple cellular spheroids that contain potential CSCs defined by morphology have been established to analyze the characteristics of OVCSCs. Although the Notch signaling pathway has been implicated in the development of chemoresistance in OVCSCs $(5,6)$, small molecules targeting OVCSCs have not yet been screened. The development of such OVCSC-specific therapies may hold the key to preventing relapse and successfully treating patients who have aggressive, non-resectable $\mathrm{OvC}$.

In cancer research, differentially expressed genes have been identified by comparing samples with and without CSCs by high-throughput technologies such as microarrays. Gene Ontology analysis of these differentially expressed genes may provide ontology terms to describe attributes in 3 biological domains, cellular component, molecular function and biological process, to interpret microarray data (7). The Kyoto Encyclopedia of Genes and Genomes (KEGG) pathway query can systemically connect differentially expressed genes with other known information on the molecular interaction networks, especially in signaling pathways (8). Also, the Connectivity Map (CMAP), a massive repository of gene expression data, provides information on changes in gene expression in several cell lines when treated with $>1,000$ bioactive compounds $(9,10)$. With these differentially expressed genes as query signatures of CSCs, the CMAP provides a novel resource to systematically screen for small molecules targeting CSC-specific genes.

In the present study, we used the CMAP to conduct a comprehensive analysis of multiple samples derived from the National Center for Biotechnology Information (NCBI) Gene Expression Omnibus (GEO) database to reveal several key pathways and OVCSC signature genes. Data on signature genes were then used to perform an OVCSC-specific drug prescreening based on co-expression extrapolation and predicted several small molecules with potential anti-OVCSC pharmacological properties, which may aid the development of OVCSC-specific drugs.

\section{Materials and methods}

Sources of whole-genome expression profiles of ovarian cancer. In the present study, we reanalyzed previously published raw data (Table I). Expression profiles of normal ovarian surface epithelium cells and ovarian cancer cells from patients with advanced disease were compared. The original CEL files by Rizzo et al (11) (GSE25191), Vathipadiekal et al (12)(GSE33874) and Wang et al (13) (GSE28799) were retrieved from the NCBI GEO database for OVCSC analysis. In brief, SPs of OvC were isolated from the serous epithelial OvC cell line IGROV1 and patient-derived ascites. Multiple cellular spheroids were isolated from the OVCAR-3 cell line on the basis of morphologic characteristics. RNA was extracted from all specimens that had OVCSC features and from non-stemness controls. Extracted RNA was pre-amplified, conjugated with fluorescent markers or biotin-labeled markers, and hybridized to an expression microarray chip. Raw data from Bonome et al (14) (GSE26712) were used to calibrate the OVCSC signature for drug prescreening. Serous epithelial OvC specimens were obtained independently by optimal debulking surgery from patients with previously untreated late-stage (III-IV) high-

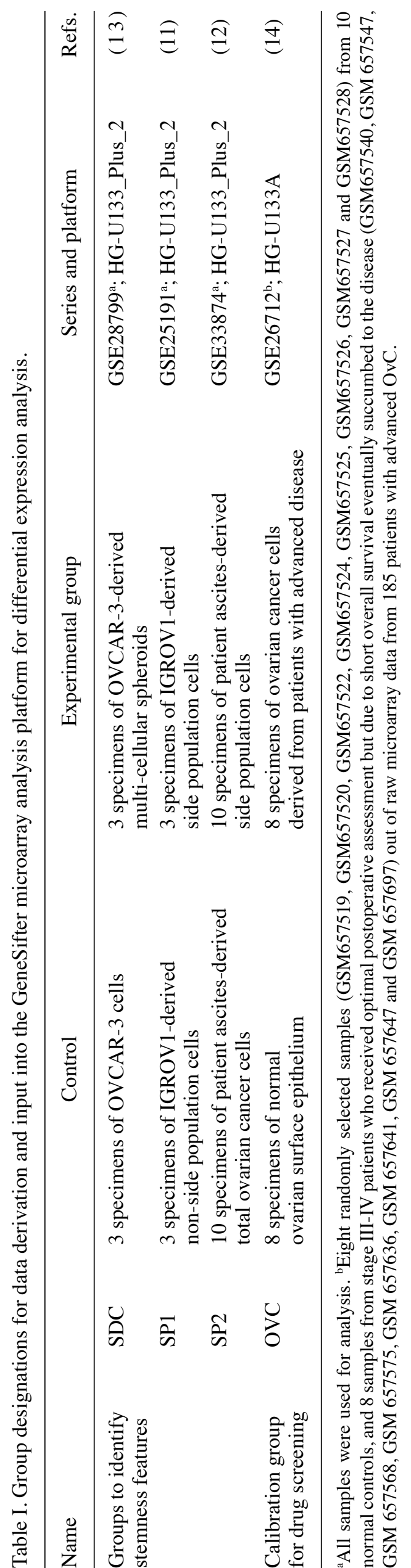


grade $(2,3)$ OvC. Normal ovarian surface epithelium cells were obtained by cytobrushing and RNA from these cells was independently analyzed by microarrays.

Preprocessing and normalization of array data. Four groups of raw data were normalized to probe level by using the RMA algorithm for background correction. Quantile normalization and multi-chip model mid-value fitting were performed. The final normalized data were output to the GeneSifter microarray analysis platform (http://www.geospiza.com/Products/ AnalysisEdition.shtml; Geospiza, Inc., Seattle, WA, USA) by groups after logarithmic transformation.

Analysis of differentially expressed genes. In the pair wise mode, normalized microarray data were analyzed to obtain differentially expressed probes by groups and probes were then assigned to genes. The following analysis parameters were used: statistics (t-test); correction (Benjamini and Hochberg); fold-change (lower, 1.5; upper, none); and quality options (one group must pass). The Exclude Control Probes option was chosen to output both upregulated and downregulated genes with $\mathrm{P}<0.05$.

Analysis of biological significance. Differentially expressed genes previously identified in the SDC, SP1 and SP2 groups were output to the Gene Ontology (GO) tool to determine their significance with respect to their biological processes, molecular functions and cellular components. These genes were also output to the KEGG pathway mapping tool to determine commonly enriched pathways. The $\mathrm{Z}$ score was used to test enrichment of GO terms and KEGG pathways in the differentially expressed gene list. A $\mathrm{Z}$ score of $>2$ or $<-2$ was considered statistically significant.

CMAP drug screening with cancer stem cell signature. To converge differentially expressed genes with ovarian cancer stem cell characteristics, analysis was performed by integrating upregulated and downregulated probes from each OVCSC group (SDC, SP1 and SP2) calibrated with the OvC group in the Venny tool, commonly upregulated and downregulated genes in OVCSC groups were identified in the following combinations: OVC/SDC + OVC/SP1 + OVC/SP2 $+\mathrm{OVC} / \mathrm{SDC} / \mathrm{SP} 1+\mathrm{OVC} / \mathrm{SDC} / \mathrm{SP} 2+\mathrm{OVC} / \mathrm{SP} 1 / \mathrm{SP} 2+\mathrm{OVC} /$ SDC/SP1/SP2.

Calibration groups were introduced for drug prescreening for necessity and sufficiency:

Necessity. The CMAP database was constructed by using thousands of HG-U133A (low-density array) expression data, but in the present study all OVCSC-related signatures were derived from HG-U133_Plus_2 (high-density array). If a low-density-based filtering is not performed, the OVCSC signature will not be compatible for CMAP querying. In addition, this filtering may reduce the accuracy or significance of differentially expressed signatures for cancer stemness groups compared with that for non-stemness controls, but there were almost no intentional bias between these groups.

Sufficiency. If a calibration group with differentially expressed genes for normal ovarian surface epithelium with respect to OVCSCs containing ovarian cancer cells derived from patients with advanced disease is not introduced, the query signature will only represent massively proliferating non-stemness cancer cells over CSCs in the CAMP screening. As the small molecules with the potential of reversing the expression signature may activate CSCs by turning them into highly proliferating cancer cells, this calibration group was introduced.

CMAP drug screening by the COXEN method with the ovarian cancer stem cell signature. By using the input of OVCSCspecific query signature genes, a preliminary list of anti-OVCSC small molecules was obtained. To further screen the potential drugs targeting OVCSCs, more stringent criteria were applied by restricting the number of repeat experiments to $>3$ times, selecting molecules with negative enrichment score (representing the potential effectiveness), using $\mathrm{P}<0.01$ for statistical tests, and setting the proportion of effective rate to $>50 \%$.

\section{Results}

Differentially expressed genes in OVCSC cells. By using the GeneSifter software, differentially expressed genes with a fold-change $>1.5$ compared with non-stemness controls and $\mathrm{P}<0.05$ was identified for each OVCSC group. Compared with non-stemness controls, there were 6,495 (3,252 upregulated and 3,243 downregulated), 1,347 (765 upregulated and 582 downregulated) and 509 (44 upregulated and 465 downregulated) differentially expressed genes in the SDC, SP1 and SP2 groups, respectively. NAB1 (NGFI-A binding protein 1) and NPIPL1 (nuclear pore complex interacting protein-like 1) were commonly upregulated in SDC and SP1. PROS1 (protein S $\alpha$ ), GREB1 (growth regulation by estrogen in breast cancer 1), PLCL1 (phospholipase C-like 1), MTUS1 (mitochondrial tumor suppressor 1), PPM1D (protein phosphatase 1D magnesium-dependent), CDC42EP3 (CDC42 effector protein Rho GTPase binding 3) and AMPD (adenosine monophosphate deaminase isoform E) were commonly downregulated in all OVCSC groups.

Analysis of biological significance. Enrichment analysis of differentially expressed genes revealed that 2,052, 1,005 and 1,581 biological processes were enriched in cells from SDC, SP1 and SP2 groups, respectively, as compared with non-stemness controls. Some biological processes were enriched in all OVCSC groups, such as tolerance induction, cell cycle regulation, stemness maintenance and anti-apoptosis (Table II). Distribution patterns of the involved biological processes, cellular components, and molecular functions were similar among the 3 groups (Fig. 1).

Enrichment analysis also revealed that 218, 137 and 99 cellular components were enriched in SDC, SP1 and SP2 groups, respectively. Cellular components such as membrane structures of drug resistance components, cell division components, and cell adhesion structures were enriched in all 3 groups (Table III).

In total, 597, 315 and 253 molecular functions were enriched in cells from SDC, SP1 and SP2 groups, respectively. Commonly enriched molecular functions in OVCSC cells included chemorepellent activity, growth factor receptor activity, epigenetic molecular functions and kinase activity (Table IV). 
Table II. Enriched and shared biological processes from commonly upregulated or downregulated genes by 2 or 3 stemness groups.

\begin{tabular}{llllll}
\hline Biological process & SDC & SP1 & SP2
\end{tabular}

Enriched by upregulated genes

Histone H3-K27 demethylation

$4.12 \quad 4.31$

Histone H4-K20 demethylation

$4.12 \quad 4.31$

Regulation of phosphatidylinositol 3-kinase activity

$4.02 \quad 2$

Histone H3-K9 demethylation

$3.64 \quad 4.09$

Negative regulation of insulin-like growth factor receptor signaling pathway

$3.61 \quad 2.51$

Tolerance induction

$3.04 \quad 3.56$

Negative regulation of BMP signaling pathway by extracellular sequestering of BMP

$2.92 \quad 6.26$

Positive regulation of tolerance induction

$2.79 \quad 2$

Regulation of tolerance induction

Organ formation

$2.74 \quad 2.2$

Response to growth factor stimulus

$2.73 \quad 2.28$

Histone lysine demethylation

$2.2 \quad 2.83$

Myoblast cell fate commitment

$2.15 \quad 2.51$

Histone H3-K36 demethylation

$2.15 \quad 2.51$

Positive regulation of T-cell tolerance induction

$2.15 \quad 2.51$

Smooth muscle tissue development

Bone development

Regulation of fat cell differentiation

Regulation of Wnt receptor signaling pathway

Negative regulation of response to stimulus

Regulation of canonical Wnt receptor signaling pathway

2.1

Regulation of BMP signaling pathway

Negative regulation of BMP signaling pathway

BMP signaling pathway

Regulation of transforming growth factor $\beta$ receptor signaling pathway

Enriched by downregulated genes

Embryo development

Negative regulation of transferase activity

Positive regulation of gene expression

Progesterone receptor signaling pathway

Regulation of toll-like receptor 3 signaling pathway

Regulation of transcription

Regulation of transcription from RNA polymerase II promoter

RNA polymerase II transcriptional preinitiation complex assembly

Epithelial cell maturation

Endodermal digestive tract morphogenesis

Negative regulation of tyrosine phosphorylation of STAT protein

Cell differentiation 
Table II. Continued.

Biological process

SDC SP1 SP2

Enriched by downregulated genes

Epithelial cell differentiation involved in prostate gland development

4.83

6.2

Wnt receptor signaling pathway

4.76

3.03

Chemotaxis

Positive regulation of Wnt receptor signaling pathway

Inactivation of MAPK activity

Apoptosis in bone marrow

Regulation of apoptosis in bone marrow

Positive regulation of cell proliferation

Negative regulation of MAP kinase activity

Negative regulation of protein serine/threonine kinase activity

Cellular response to stimulus

Positive regulation of cell cycle

Cell motility

Regulation of response to stimulus

Positive regulation of response to stimulus

Apoptotic mitochondrial changes

Cell-cell adhesion

Regulation of developmental process

Positive regulation of release of cytochrome $c$ from mitochondria

Epithelial cell differentiation

Release of cytochrome $c$ from mitochondria

Urogenital system development

Positive regulation of mitotic cell cycle

Fibroblast growth factor receptor signaling pathway involved in positive regulation of cell proliferation

Negative regulation of CD40 signaling pathway

Negative regulation of toll-like receptor 3 signaling pathway

Regulation of CD40 signaling pathway

Regulation of release of cytochrome $c$ from mitochondria

Response to stimulus

Response to external stimulus

Glandular epithelial cell differentiation

Regulation of MAP kinase activity

Response to chemical stimulus

Epithelial cell development

Fibroblast migration

Regulation of S phase

Apoptosis

Cell proliferation

Regulation of cell proliferation

Epithelium development

Programmed cell death

Regulation of cell differentiation

Cell death

Regulation of binding

Cell-cell adhesion mediated by integrin

MAPKKK cascade

Regulation of cell migration

Morphogenesis of an epithelium

Regulation of cell motility

Epithelial cell differentiation involved in mammary gland alveolus development 
Table II. Continued.

\begin{tabular}{|c|c|c|c|}
\hline Biological process & SDC & SP1 & SP2 \\
\hline \multicolumn{4}{|l|}{ Enriched by downregulated genes } \\
\hline Developmental maturation & & 3.23 & 2.2 \\
\hline Erythrocyte differentiation & & 3.16 & 3.89 \\
\hline Regulation of toll-like receptor 4 signaling pathway & & 2.84 & 5.67 \\
\hline Positive regulation of focal adhesion assembly & & 2.84 & 2.67 \\
\hline Positive regulation of macrophage differentiation & & 2.84 & 2.67 \\
\hline Cellular response to transforming growth factor $\beta$ stimulus & & 2.58 & 2.42 \\
\hline Regulation of histone modification & & 2.44 & 2.44 \\
\hline Cell maturation & & 2.44 & 2.21 \\
\hline Response to transforming growth factor $\beta$ stimulus & & 2.37 & 2.21 \\
\hline Regulation of epithelial to mesenchymal transition & & 2.23 & 3.44 \\
\hline Lymph vessel development & & 2.19 & 2.04 \\
\hline Regulation of histone methylation & & 2.19 & 4.49 \\
\hline Regulation of myeloid cell differentiation & & 2.18 & 3.56 \\
\hline Response to growth factor stimulus & & 2.16 & 2.95 \\
\hline Negative regulation of histone modification & & 2.03 & 4.22 \\
\hline
\end{tabular}

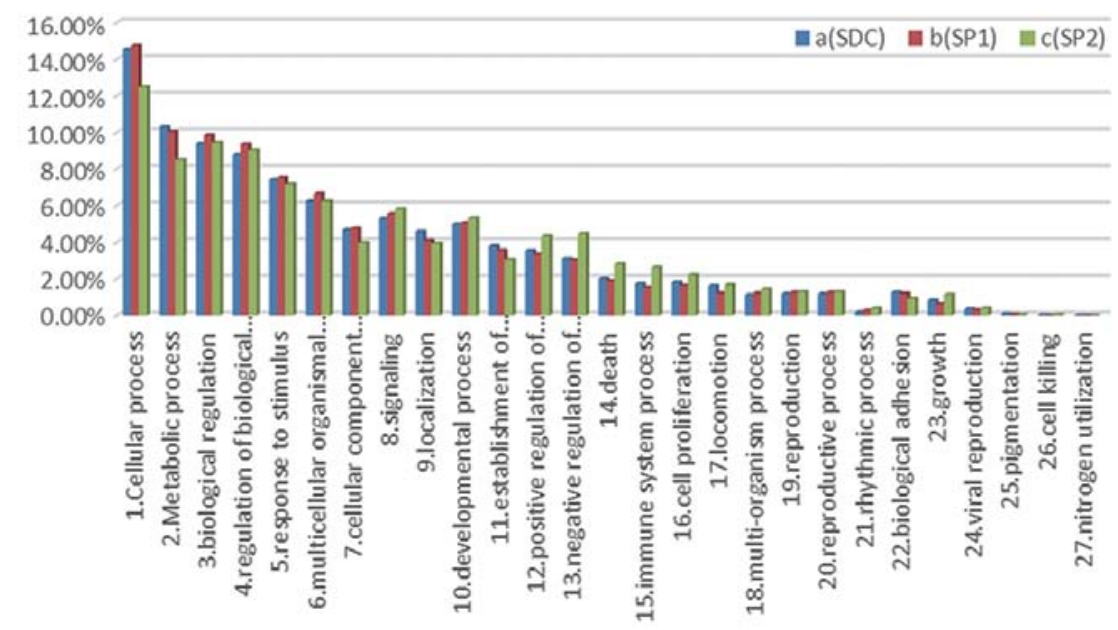

Figure 1. Distribution of the proportion of biological processes from the 3 groups of differentially expressed genes.

With regard to signaling pathways, 57, 31 and 33 KEGG pathways were enriched in cells from SDC, SP1 and SP2 groups, respectively. Key KEGG pathways such as the ErbB pathway, ECM-receptor pathway, endocytosis pathway and adherens junction pathway were enriched in all 3 groups (Table V).

Cancer stem cell signature-specific drug prescreening. The list of differentially expressed genes identified does not necessarily represent the cancerous features of OVCSCs. CSCs are highly quiescent whereas differentiated cancer cells are highly proliferative. Genes responsible for the quiescent state were also included in the above lists; however, they may not be ideal targets for therapy, as reversion of the quiescent state of OVCSCs may translate to the massive production of cancerous cells, which is undesirable. Therefore, it is necessary to focus on the cancerous features of OVCSCs. Compared with the normal ovarian surface epithelium, there were 2,669 upregulated and 3,384 downregulated genes in cells derived from patients with advanced OvC (Fig. 2). This set of genes represented cancerous features, and these genes were considered candidates for the OVCSC signature. With this calibration set, genes unrelated to the cancerous features of OVCSCs, such as those responsible for the quiescent state, were removed from the list of differentially expressed genes. Compared with non-stemness controls, there were 225 upregulated and 373 downregulated probes in the SDC group, 23 upregulated and 22 downregulated probes in the SP1 group and 5 upregulated and 38 downregulated probes in the SP2 group. There were 2 shared upregulated probes 
Table III. Enriched and shared cellular components from significantly upregulated or downregulated genes by 2 or 3 stemness groups.

\begin{tabular}{|c|c|c|c|}
\hline Cellular component & SDC & SP1 & SP2 \\
\hline \multicolumn{4}{|l|}{ Enriched by upregulated genes } \\
\hline Coated membrane & 3.41 & 2.11 & \\
\hline Membrane coat & 3.41 & 2.11 & \\
\hline Plasma membrane-enriched fraction & 3.21 & 2.37 & \\
\hline 1-Phosphatidylinositol-4-phosphate 3-kinase, class IA complex & 2.96 & 6.57 & \\
\hline Basal lamina & 2.77 & 2.75 & \\
\hline Polar microtubule & 2.62 & 3.06 & \\
\hline Centrosome & & 3.15 & 2.33 \\
\hline Nuclear envelope & & 2.04 & 2.22 \\
\hline \multicolumn{4}{|l|}{ Enriched by downregulated genes } \\
\hline Apicolateral plasma membrane & 5.04 & 2.03 & 2.7 \\
\hline Apical junction complex & 4.45 & 2.09 & 2.76 \\
\hline Occluding junction & 4.43 & 2.41 & 3.13 \\
\hline Tight junction & 4.43 & 2.41 & 3.13 \\
\hline Intracellular & 4.65 & & 2.09 \\
\hline Intracellular part & 4.46 & & 2.03 \\
\hline Nucleus & 4.4 & & 4.04 \\
\hline Ruffle & 3.91 & & 2.67 \\
\hline mRNA cap binding complex & 3.4 & & 2.06 \\
\hline RNA cap binding complex & 3.4 & & 2.06 \\
\hline Plasma membrane & 2.94 & & 2.09 \\
\hline Ruffle membrane & 2.85 & & 4.04 \\
\hline Receptor complex & 2.77 & & 3.6 \\
\hline Leading edge membrane & 2.57 & & 2.54 \\
\hline Plasma membrane part & 2.45 & & 2.02 \\
\hline Junctional sarcoplasmic reticulum membrane & 2.21 & & 3.01 \\
\hline Excitatory synapse & 2.21 & & 3.01 \\
\hline $\mathrm{I}-\kappa \beta / \mathrm{NF}-\kappa \beta$ complex & 2.21 & & 3.01 \\
\hline Nuclear lumen & 2.19 & & 2.4 \\
\hline Basolateral plasma membrane & 2.12 & & 3.19 \\
\hline Neuromuscular junction & 2.08 & & 2.14 \\
\hline Synaptobrevin 2-SNAP-25-syntaxin-1a-complexin I complex & & 5.21 & 5.05 \\
\hline Synaptobrevin 2-SNAP-25-syntaxin-1a complex & & 4.18 & 4.04 \\
\hline Viral capsid & & 4.18 & 4.04 \\
\hline Virion & & 3.55 & 3.43 \\
\hline Virion part & & 3.55 & 3.43 \\
\hline Apical part of cell & & 2.73 & 2.03 \\
\hline Golgi lumen & & 2.46 & 2.35 \\
\hline
\end{tabular}

and 6 shared downregulated probes between cells from SP1 and SDC groups; 7 shared downregulated probes between cells from the SDC and SP2 groups, and 1 commonly downregulated probe between cells from the SP1 and SP2 groups compared with non-stemness controls (Fig. 3; Table VI). An OVCSC signature was generated by combining the 255 upregulated tags $(23+2+225+5)$ and the 447 downregulated tags $(22+6+373+7+38+1)$.

OVCSC signatures of 255 upregulated probes and 447 downregulated probes were analyzed by the CMAP for a drug prescreening. Of the 6,100 proceeded instances, it was predicted that 1,500 had the potential to promote the OVCSC signature, as indicated by their positive enrichment scores, whereas 1,419 could have anti-OVCSC effects, as reflected by their negative enrichment scores. By further filtering the 1,419 molecules with negative scores with the described criteria, 18 remained as the most promising therapeutic small-molecule candidates to target OVCSCs, for example SC-560, disulfiram (DS), thapsigargin, esculetin, cinchonine, alvespimycin and tanespimycin (Table VII). 
Table IV. Enriched and shared molecular functions from significantly upregulated or downregulated genes by 2 or 3 stemness groups.

Molecular function

Enriched by upregulated genes

Inositol 1,4,5-trisphosphate-sensitive calcium-release channel activity

Dioxygenase activity

Oxidoreductase activity, acting on single donors with incorporation of molecular oxygen

Oxidoreductase activity, acting on single donors with incorporation of molecular oxygen, incorporation of 2

Inositol-1,4,5-trisphosphate receptor activity

Androsterone dehydrogenase activity

trans-1,2-dihydrobenzene-1,2-diol dehydrogenase activity

Androsterone dehydrogenase (A-specific) activity

Glutaminase activity

Histone demethylase activity (H3-K27 specific)

Histone demethylase activity (H4-K20 specific)

Oxidoreductase activity, acting on paired donors, with incorporation or reduction

of molecular oxygen, 2-oxogl

Histone demethylase activity (H3-K9 specific)

Metal ion binding

L-ascorbic acid binding

Protein binding

Calcium-dependent cysteine-type endopeptidase inhibitor activity

Poly(G) RNA binding

Poly-glutamine tract binding

Chemorepellent activity

Insulin binding

S100 $\beta$ binding

Protein tyrosine kinase activity

Bile acid binding

Platelet-derived growth factor receptor binding

Transmembrane receptor protein kinase activity

Transmembrane receptor protein tyrosine kinase activity

Virion binding

Receptor tyrosine kinase binding

Enriched by downregulated genes

Fibroblast growth factor receptor activity

Protein anchor

Thiamine transmembrane transporter activity

Thiamine uptake transmembrane transporter activity

Uptake transmembrane transporter activity

Reduced folate carrier activity

General transcriptional repressor activity

Protein serine/threonine phosphatase inhibitor activity

Phosphatidylserine binding

Heparan sulfate proteoglycan binding

Interleukin-1, type II, blocking receptor activity

Epidermal growth factor receptor activity

Oncostatin-m receptor activity

Epinephrine binding

$\beta 2$-adrenergic receptor activity

Calcium-dependent protein kinase c activity

Calcium-dependent protein serine/threonine kinase activity
SDC

SP1 SP2

5.12

3.49

4.41

4.48

4.41

4.48

4.41

4.48

4.26

2.94

4.26

2.94

4.26

2.94

4.18

4.38

$4.18 \quad 4.38$

$4.18 \quad 4.38$

$4.18 \quad 4.38$

$3.9 \quad 3.33$

3.66

2.56

3.57

2.09

3.48

2.12

$3.13 \quad 2.66$

$2.95 \quad 6.36$

$2.95 \quad 6.36$

2.95

6.36

2.61

2.94

2.61

2.94

2.61

2.94

2.24

3.18

2.19

2.56

2.09

4.44

4.45

2.13

3.33

2.27

2.5

2.04

4.72

3.67

3.14

3

3.22

4.21

$4.19 \quad 5.25$

$4.19 \quad 5.25$

$4.19 \quad 5.25$

$2.62 \quad 3.58$

$2.29 \quad 4.73$

$2.19 \quad 3.14$

$2.06 \quad 2.01$

2.06

2.01

4.19 
Table IV. Continued.

Molecular function

SDC

SP1

SP2

Enriched by downregulated genes

Cytoskeletal regulatory protein binding

2.96

7.25

Endoribonuclease activity, cleaving siRNA-paired mRNA

2.96

7.25

GTP cyclohydrolase activity

2.96

7.25

GTP cyclohydrolase i activity

2.96

7.25

Protein channel activity

2.96

7.25

Interleukin-1 binding

2.86

4

Interleukin-1 receptor activity

2.85

5.22

RNA polymerase II transcription factor binding

2.85

2.43

Sodium channel regulator activity

2.85

2.43

Actin filament binding

2.84

2.97

Protein tyrosine kinase activity

2.82

2.19

Adenylate cyclase binding

2.62

3.42

Norepinephrine binding

2.62

3.42

Insulin receptor binding

2.58

2.07

Transmembrane receptor protein tyrosine kinase activity

2.54

2.53

Co-SMAD binding

2.54

2.22

RNA cap binding

2.54

2.22

Small conjugating protein ligase activity

2.51

2.81

Map kinase tyrosine/serine/threonine phosphatase activity

2.44

3.6

Protein dimerization activity

2.41

3.03

Growth factor binding

Ubiquitin thiolesterase activity

Acid-amino acid ligase activity

Transcription activator activity

Transmembrane receptor protein kinase activity

Adrenergic receptor activity

Map kinase phosphatase activity

Ligase activity, forming carbon-nitrogen bonds

Cyclohydrolase activity

Prostaglandin e receptor activity

Protein serine/threonine kinase activator activity

2.19

2.19

3

Transforming growth factor $\beta$ receptor, pathway-specific cytoplasmic mediator activity

2.01

Ubiquitin-protein ligase activity

A1 adenosine receptor binding

Retinoic acid binding

AMP deaminase activity

Phosphatidylinositol-4-phosphate 3-kinase activity

Adenosine receptor binding

$\mathrm{N}$-acetylglucosamine 6- $\mathrm{O}$-sulfotransferase activity

Wnt-protein binding

Thyroid hormone receptor activity

Transcription regulator activity

Cation:chloride symporter activity

Monovalent cation:hydrogen antiporter activity

Syntaxin binding

Transcription repressor activity 
Table V. The shared KEGG pathways of differentially expressed genes in ovarian cancer stemness groups.

\begin{tabular}{|c|c|c|c|}
\hline $\mathrm{SDC}, \mathrm{SP} 1$ and SP2 & SDC and SP1 & $\mathrm{SDC}$ and SP2 & SP1 and SP2 \\
\hline $\begin{array}{l}\text { ErbB signaling } \\
\text { pathway }\end{array}$ & Metabolic pathways & Pathways in cancer & T-cell receptor signaling pathway \\
\hline \multirow[t]{11}{*}{ Prostate cancer } & Focal adhesion & Endocytosis & Amoebiasis \\
\hline & ECM-receptor interaction & MAPK signaling pathway & NOD-like receptor signaling pathway \\
\hline & Dilated cardiomyopathy & Adherens junction & Dorso-ventral axis formation \\
\hline & Hypertrophic cardiomyopathy & $\begin{array}{l}\text { Epithelial cell signaling } \\
\text { in Helicobacter pylori infection }\end{array}$ & Lysine biosynthesis \\
\hline & Small-cell lung cancer & B-cell-receptor signaling pathway & \\
\hline & $\begin{array}{l}\text { Arrhythmogenic right } \\
\text { ventricular cardiomyopathy }\end{array}$ & Hedgehog signaling pathway & \\
\hline & Peroxisome & $\begin{array}{l}\text { Pathogenic Escherichia coli } \\
\text { infection }\end{array}$ & \\
\hline & Systemic lupus erythematosus & & \\
\hline & $\begin{array}{l}\text { Glycosaminoglycan } \\
\text { biosynthesis-keratan sulfate }\end{array}$ & & \\
\hline & $\begin{array}{l}\text { Glycosylphosphatidylinositol } \\
\text { (GPI)-anchor biosynthesis }\end{array}$ & & \\
\hline & $\begin{array}{l}\text { D-Glutamine and D-glutamate } \\
\text { metabolism }\end{array}$ & & \\
\hline
\end{tabular}

\section{Discussion}

OvC has the highest mortality rates of all gynecological malignancies and the 5-year survival rates of patients with $\mathrm{OvC}$ remain poor (15). CSC theory can consistently illustrate many clinical and pathological features of $\mathrm{OvC}$, and they have been well supported by the isolation and identification of CSCs in side populations, multi-cellular spheroids from tumor bulk (16) and OvC cell lines (4). CSCs, which often consist of a low fraction, have been established to have a general connection to $\mathrm{OvC}$ progression, such as relapse, migration and drug resistance. Efforts have been made to study the characteristics of CSCs in detail and to effectively eliminate them.

By comparing the genome-wide expression profiles of OVCSC-specific clinical and experimental specimens from the GEO database, we identified the differentially expressed genes shared by 3 OVCSC groups. Most of the OVCSC signature genes were closely related with respect to CSC properties and OvC progression. NGFI-A binding protein 1 has been reported to be upregulated in several OVCSC groups (17). By inhibiting the transcription factor EGR-1, NAB1 downregulates the transcription of aminolevulinic acid synthase 1 (ALAS1), leading to increase in the intracellular level of heme via feedback regulation (17). High levels of heme, together with iron-mediated oxidative stress and inflammation in patients with endometriosis, can accelerate the progression of OvC (18). In the present study, genes that were downregulated in all OVCSC groups included PROS1 (protein S $\alpha$ ), GREB1, KLF9 (Kruppel like factor 9), MTUS1 (mitochondrial tumor suppressor 1), PPM1D (protein phosphatase 1D magnesium-dependent) and inhibitor of DNA binding 2 (ID2). The downregulation of gene PROS1, a member of the HNF4 $\alpha$ tumor suppressor network, can promote cell proliferation and finally leads to tumor progression (19). In breast cancer, estrogen-receptor-negative cancer cells with low levels of GREB1 expression (20) have more stem cell features than other cancer cells (21). Overexpression of KLF9, a differentiation-related transcription factor, promoted differentiation of malignant glioma stem cell spheres and inhibited their proliferation in vivo in a xenotransplantation model (22). The downregulation of KLF9 in OVCSCs suggests that its absence may be required to maintain stemness. We also confirmed downregulation of the potential tumor suppressor gene MTUS1 in OVCSCs, which is consistent with a previous study showing that downregulation of MTUS1 and Claudin-1 (CL-1) in various human tumors is related to active proliferation, poor differentiation and poor clinical outcomes (23). PPM1D, a highly expressed oncogene in various human tumors, can inactivate CHK1 and p53 via dephosphorylation, leading to cisplatin resistance in cancer cells. Therefore, this gene is a potential target for treatment of OvC (24,25). ID2 is a new drug target, as high expression of ID2 can maintain the pluripotency of neural stem cell spheroids by direct inhibition of p53 (26). However, both PPM1D and ID2 were downregulated in the OVCSC groups, which may be due to the concealment and quiescence of CSCs, but this requires further investigation.

To identify the CSC features shared by 3 OVCSC groups, detailed Gene Ontology analysis was performed by using the GeneSifter software using domains of biological processes, cellular components and molecular functions. Differentially expressed genes were significantly enriched in biological features such as tolerance induction, cell cycle regulation, stemness maintenance and anti-apoptosis (all processes involved in OvC progression), which can easily distinguish OVCSCs from other OvC cells. By analyzing the enriched signaling pathways involving differentially expressed genes, we found that OVCSCs were different from other $\mathrm{OvC}$ cells in the ECM-receptor pathway, focal adhesion pathway and adherens junction 

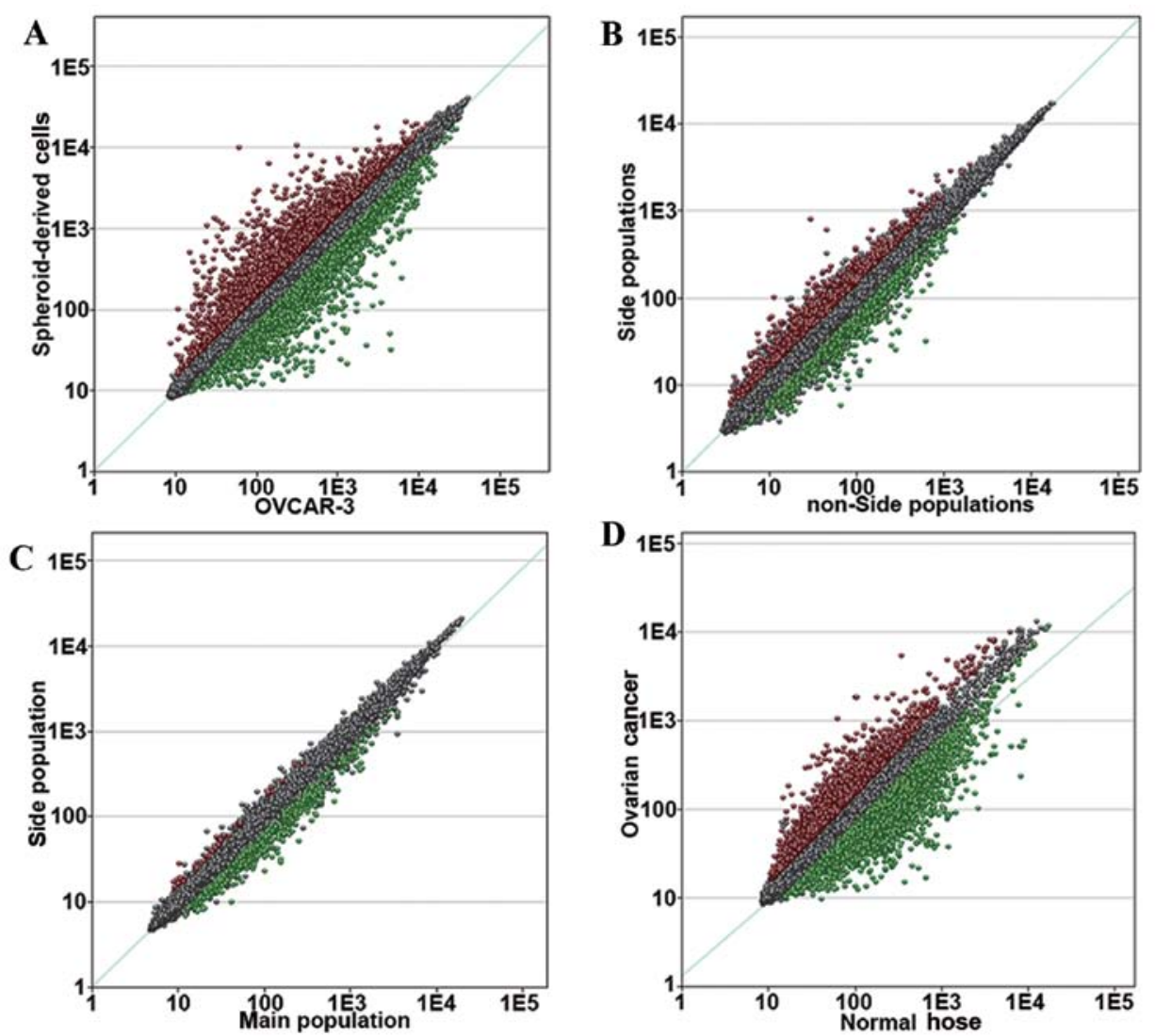

Figure 2. Scatterplot of differentially expressed genes in the (A) SDC group, (B) SP1 group, (C) SP2 group and (D) OVC group was used for drug screening calibration only. Each dot of the scatter plot represents the signal intensity of chip hybridization for every expressed gene. Those genes that neither have inconspicuous expression profiles nor match previously described criteria are represented by gray dots around or on the diagonal. Genes that have significant differential expression profiles are represented by red or green dots for upregulation or downregulation features, respectively, and are scattered far from the slash.
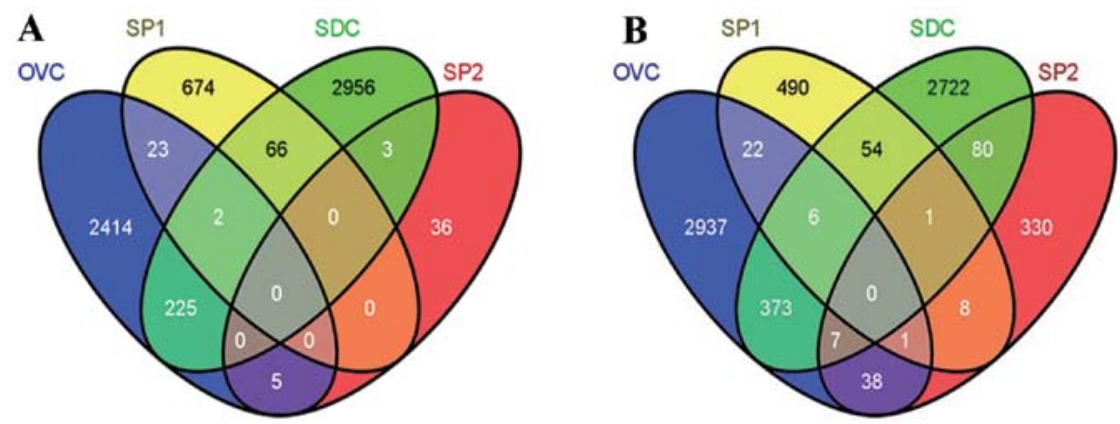

Figure 3. Shared probe numbers for (A) upregulated and (B) downregulated genes that were statistically significant among ovarian cancer stem cell groups and the calibration group.

signaling pathway. This finding is consistent with findings from other studies showing a strong correlation between these signaling pathways and epithelial stem cell proliferation, cancer invasion and migration and staged tumor progression $(27,28)$. Moreover, some of these pathways have important functions in CSCs. The ErbB signaling pathway mediates epithelialmesenchymal transitions in breast cancer, and is enriched in CD $44^{+} / \mathrm{CD} 24^{-}$breast CSCs (29-31). During Helicobacter pylori infection, normal gastrointestinal stem cells are disrupted through epithelial cell signaling and the downstream STAT and WNT signaling pathways, leading to the genesis and progression of gastrointestinal tumors (32). These signaling pathways may also cooperate in colonic glands epithelial tumor stem cells. Pathogenic Escherichia coli infection correlates with the formation and progression of an epithelial colonic tumor, indicating that the same pathway is also active in OVCSCs (33). Endocytosis-related pathways are enriched in OVCSCs and represent endocytosis mediated by OVCSC-specific surface markers such as CD133 and CD44 (34).

Following characterization of signature genes in OVCSCs, co-expression extrapolation was performed with the CMAP, and small-molecule compounds with potential anti-OVCSC pharmacological properties were identified. Notably, some of these compounds [such as SC-560, disulfiram (DS), thapsigargin, esculetin, cinchonine, alvespimycin and tanespimycin] have been tested in other tumors. As a selective inhibitor of 


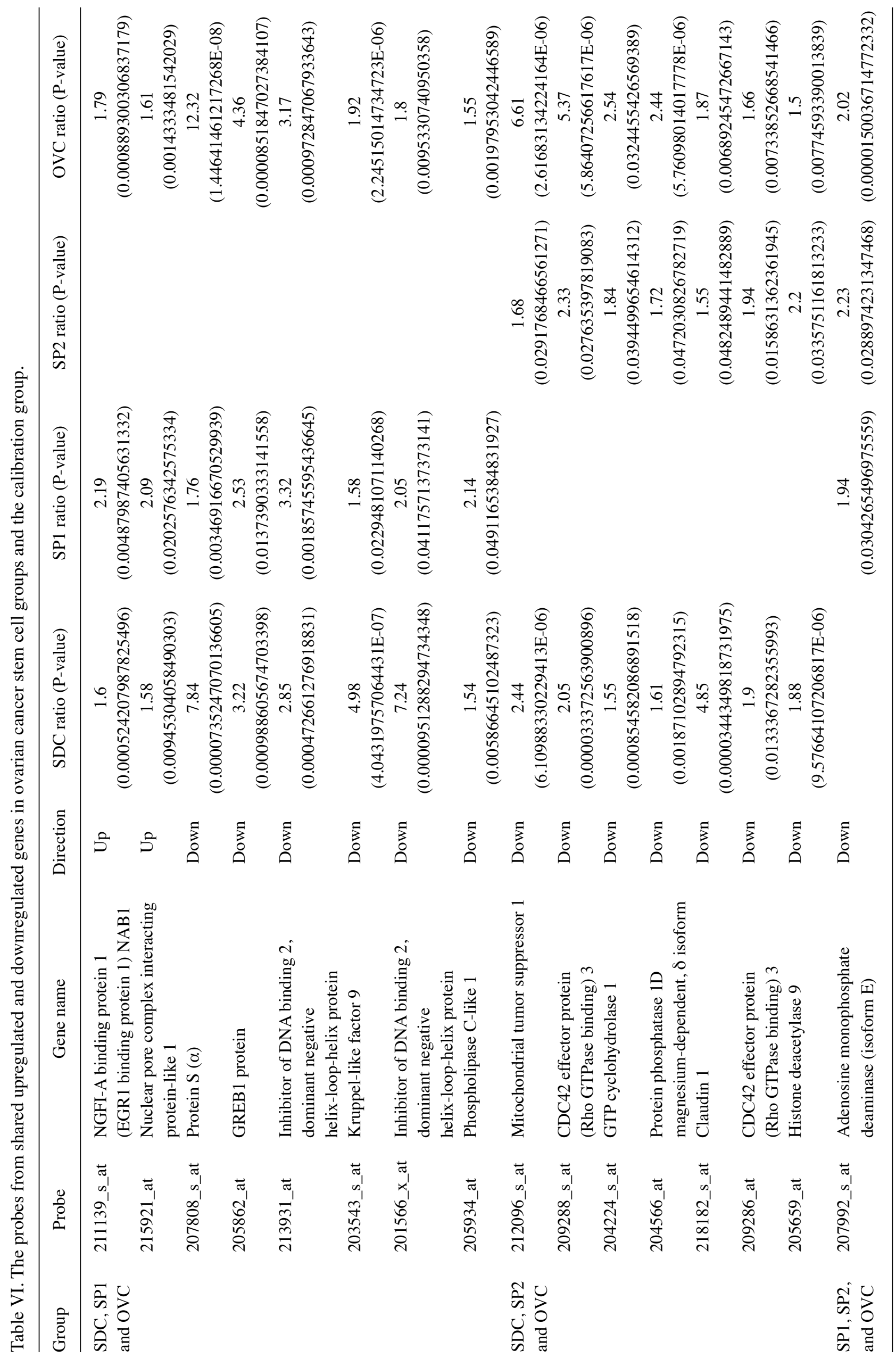


Table VII. Eighteen therapeutic small-molecule drugs with potential OVCSC-specific targeting abilities in ovarian cancer.

\begin{tabular}{|c|c|c|c|c|c|c|c|}
\hline $\begin{array}{l}\text { Connectivity Map } \\
\text { name }\end{array}$ & Mean & $\mathrm{N}$ & Enrichment & P-value & Specificity & $\%$ Non-null & Category \\
\hline Sc-560 & -0.52 & 3 & -0.927 & 0.00062 & 0.0121 & 100 & COX-1 inhibitor \\
\hline Prestwick-1082 & -0.43 & 3 & -0.899 & 0.00192 & 0.0246 & 100 & \\
\hline Puromycin & -0.48 & 4 & -0.865 & 0.00062 & 0.0373 & 100 & Protein synthesis inhibitor \\
\hline Doxylamine & -0.47 & 5 & -0.854 & 0.00016 & 0.016 & 100 & Antihistamines \\
\hline Pralidoxime & -0.36 & 4 & -0.848 & 0.00097 & 0 & 100 & Cholinesterase reactivator \\
\hline Disulfiram & -0.4 & 5 & -0.846 & 0.00024 & 0.0072 & 100 & Proteasome inhibitor \\
\hline Thapsigargin & -0.34 & 3 & -0.841 & 0.00809 & 0.1161 & 100 & Non-competitive SERCA inhibitor \\
\hline Esculetin & -0.32 & 3 & -0.836 & 0.00877 & 0 & 100 & \\
\hline Phenazone & -0.39 & 3 & -0.833 & 0.00931 & 0.0231 & 100 & Analgesic and antipyretic \\
\hline Cinchonine & -0.51 & 4 & -0.787 & 0.00416 & 0.0617 & 100 & Alkaloid \\
\hline Lycorine & -0.29 & 5 & -0.762 & 0.00144 & 0.08 & 80 & Protein synthesis inhibitor \\
\hline Benzthiazide & -0.17 & 4 & -0.756 & 0.00716 & 0.0204 & 50 & Diuretic and antihypertensive \\
\hline Naltrexone & -0.22 & 5 & -0.735 & 0.0027 & 0.0225 & 60 & Opioid receptor antagonist \\
\hline Atropine oxide & -0.21 & 5 & -0.714 & 0.00417 & 0.0062 & 60 & \\
\hline Pyrimethamine & -0.28 & 5 & -0.703 & 0.00505 & 0.02 & 80 & DHFR inhibitor \\
\hline Diethylstilbestrol & -0.27 & 6 & -0.641 & 0.00612 & 0.0164 & 83 & Synthetic nonsteroidal estrogen \\
\hline Alvespimycin & -0.24 & 12 & -0.501 & 0.00255 & 0.0797 & 58 & Hsp90 inhibitor \\
\hline Tanespimycin & -0.19 & 62 & -0.482 & 0 & 0.0672 & 53 & Hsp90 inhibitor \\
\hline
\end{tabular}

cyclooxygenase-1, SC-560 significantly inhibits cell proliferation and arrests cells in the G0/G1 phase. SC-560 can induce autophagy of colon cancer cells in vitro (35) and SC-560 effectively suppresses tumor growth in animals xenografted with the ovarian cancer cell line SKOV-3 by inhibiting cell proliferation and promoting apoptosis (36). Taken together, our results and those from previous studies on OVCSCs suggest that SC-560 is a promising target for $\mathrm{OvC}$ treatment. The presence of the recently identified CSC marker aldehyde dehydrogenase (ALDH) has been validated in many solid tumors, including breast, colon cancer and OvC (37). Disulfiram (DS), an ALDH protease inhibitor, might suppress the migration of glioma stem cells and be used as adjuvant treatment after resection and chemotherapy (38). A recent study confirmed that DS effectively inhibits the formation of breast cancer stem cell spheres while promoting the cytotoxic effect of taxol on breast cancer cell lines by simultaneously inducing reactive oxygen species and inhibiting the NF- $\kappa \mathrm{B}$ signaling pathway. Thapsigargin has been tested in clinical trials for its high efficacy in targeting CSC-specific signaling pathways (39). The natural compound esculetin effectively inhibits Ras/ERK1/2-mediated in vitro proliferation of colon cancer cells (40). In vivo studies also indicate that esculetin can significantly enhance the chemotherapy effects of cisplatin by regulating the expression of p53/Akt/phosphatase while reducing side-effects such as induced nephrotoxicity and acute leucopenia (41). The alkaloid cinchonine may reverse multi-drug resistance (MDR), which is an important mechanism for chemotherapy failure. In 2001, Furusawa et al (42) found that cinchonine enhances doxorubicin-induced apoptosis in multi-drug-resistant P388 leukemia cells. A recent study on cervical cancer confirmed that cinchonine not only reverses the multi-drug-resistant properties of tumor cells but also has synergistic effects on taxol-induced apoptosis (43). Therefore, cinchonine may also help overcome MDR in OVCSCs. It has recently been shown that the opioid antagonist naltrexone (NTX) effectively inhibits the proliferation of $\mathrm{OvC}$ in vitro and in xenograft tumor models. NTX can significantly enhance the efficacy of cisplatin and effectively inhibit tumor progression while alleviating the cytotoxic effects of chemotherapy (44). The heat shock protein 90 (Hsp90) inhibitors alvespimycin and tanespimycin have synergistic and sensitizing effects with cisplatin and other chemotherapy drugs used to treat breast, bladder cancer, and nervous gliomas via their specific antiCSC effects (45-47). In clinical trials, several Hsp90 inhibitors have been effective in promoting targeted therapies of $\mathrm{OvC}$. The above compounds provide new options to avoiding chemotherapy failure by specifically targeting CSCs (48). In the present study, we compared gene expression profiles from several OVCSC samples with their non-stemness cancer controls. Our study revealed that OVCSCs related differentially expressed genes, enriched Gene Ontology properties and key signaling pathways, and generated an OVCSC-specific signature for screening the small-molecule compounds with potential anti-OVCSC pharmacological properties. Thus, this approach may provide new insights into developing specific drugs that target OVCSCs.

\section{Acknowledgements}

The present study was supported by the Youth Program of Tianjin Nature Science Foundation (no. 13JCQNJC10700), the Tianjin Technology Support Program of International Science and Technology Cooperation (09ZCZDSF03800) and the 973 Program (Grant 2009CB918903). 


\section{References}

1. Dingli D and Michor F: Successful therapy must eradicate cancer stem cells. Stem Cells 24: 2603-2610, 2006.

2. Frank NY, Schatton T and Frank MH: The therapeutic promise of the cancer stem cell concept. J Clin Invest 120: 41-50, 2010.

3. Clarke-Pearson DL: Clinical practice. Screening for ovarian cancer. N Engl J Med 361: 170-177, 2009.

4. Zhang S, Balch C, Chan MW, et al: Identification and characterization of ovarian cancer-initiating cells from primary human tumors. Cancer Res 68: 4311-4320, 2008.

5. Rahman MT, Nakayama K, Rahman M, et al: Notch3 overexpression as potential therapeutic target in advanced stage chemoresistant ovarian cancer. Am J Clin Pathol 138: 535-544, 2012.

6. McAuliffe SM, Morgan SL, Wyant GA, et al: Targeting Notch, a key pathway for ovarian cancer stem cells, sensitizes tumors to platinum therapy. Proc Natl Acad Sci USA 109: E2939-E2948, 2012.

7. Gene Ontology Consortium: The Gene Ontology project in 2008. Nucleic Acids Res 36: D440-D444, 2008.

8. Kanehisa M, Goto S, Kawashima S, Okuno Y and Hattori M The KEGG resource for deciphering the genome. Nucleic Acids Res 32: D277-D280, 2004.

9. Lamb J, Crawford ED, Peck D, et al: The Connectivity Map: using gene-expression signatures to connect small molecules, genes, and disease. Science 313: 1929-1935, 2006.

10. Lee JK, Havaleshko DM, Cho H, et al: A strategy for predicting the chemosensitivity of human cancers and its application to drug discovery. Proc Natl Acad Sci USA 104: 13086-13091, 2007.

11. Rizzo S, Hersey JM, Mellor P, et al: Ovarian cancer stem cell-like side populations are enriched following chemotherapy and overexpress EZH2. Mol Cancer Ther 10: 325-335, 2011.

12. Vathipadiekal V, Saxena D, Mok SC, Hauschka PV, Ozbun L and Birrer MJ: Identification of a potential ovarian cancer stem cell gene expression profile from advanced stage papillary serous ovarian cancer. PLoS One 7: e29079, 2012.

13. Wang L, Mezencev R, Bowen NJ, Matyunina LV and McDonald JF: Isolation and characterization of stem-like cells from a human ovarian cancer cell line. Mol Cell Biochem 363: 257-268, 2012.

14. Bonome T, Levine DA, Shih J, et al: A gene signature predicting for survival in suboptimally debulked patients with ovarian cancer. Cancer Res 68: 5478-5486, 2008.

15. Ozols RF: Treatment goals in ovarian cancer. Int J Gynecol Cancer 15 (Suppl 1): 3-11, 2005.

16. Bapat SA, Mali AM, Koppikar CB and Kurrey NK: Stem and progenitor-like cells contribute to the aggressive behavior of human epithelial ovarian cancer. Cancer Res 65: 3025-3029, 2005.

17. Gotoh S, Nakamura T, Kataoka T and Taketani S: Egr-1 regulates the transcriptional repression of mouse $\delta$-aminolevulinic acid synthase 1 by heme. Gene 472: 28-36, 2011.

18. Munksgaard PS and Blaakaer J: The association between endometriosis and ovarian cancer: a review of histological, genetic and molecular alterations. Gynecol Oncol 124: 164-169, 2012.

19. Grigo K, Wirsing A, Lucas B, Klein-Hitpass L and Ryffel GU: $\mathrm{HNF} 4 \alpha$ orchestrates a set of 14 genes to down-regulate cell proliferation in kidney cells. Biol Chem 389: 179-187, 2008.

20. Hnatyszyn HJ, Liu M, Hilger A, et al: Correlation of GREB1 mRNA with protein expression in breast cancer: validation of a novel GREB1 monoclonal antibody. Breast Cancer Res Treat 122: 371-380, 2010.

21. Sahab ZJ, Man YG, Byers SW and Sang QX: Putative biomarkers and targets of estrogen receptor negative human breast cancer. Int J Mol Sci 12: 4504-4521, 2011.

22. Ying M, Sang Y, Li Y, et al: Kruppel-like family of transcription factor 9, a differentiation-associated transcription factor, suppresses Notch1 signaling and inhibits glioblastoma-initiating stem cells. Stem Cells 29: 20-31, 2011

23. Ding X, Zhang N, Cai Y, et al: Down-regulation of tumor suppressor MTUS1/ATIP is associated with enhanced proliferation, poor differentiation and poor prognosis in oral tongue squamous cell carcinoma. Mol Oncol 6: 73-80, 2012.

24. Tan DS, Lambros MB, Rayter S, et al: PPM1D is a potential therapeutic target in ovarian clear cell carcinomas. Clin Cancer Res 15: 2269-2280, 2009.

25. Ali AY, Abedini MR and Tsang BK: The oncogenic phosphatase PPM1D confers cisplatin resistance in ovarian carcinoma cells by attenuating checkpoint kinase 1 and p53 activation. Oncogene 31: 2175-2186, 2012

26. Paolella BR, Havrda MC, Mantani A, Wray CM, Zhang Z and Israel MA: p53 directly represses Id2 to inhibit the proliferation of neural progenitor cells. Stem Cells 29: 1090-1101, 2011.
27. Krupp M, Maass T, Marquardt JU, et al: The functional cancer map: a systems-level synopsis of genetic deregulation in cancer. BMC Med Genomics 4: 53, 2011.

28. Lane D, Goncharenko-Khaider N, Rancourt C and Piche A: Ovarian cancer ascites protects from TRAIL-induced cell death through $\alpha v \beta 5$ integrin-mediated focal adhesion kinase and Akt activation. Oncogene 29: 3519-3531, 2010.

29. Nakanishi T, Chumsri S, Khakpour N, et al: Side-population cells in luminal-type breast cancer have tumour-initiating cell properties, and are regulated by HER 2 expression and signalling. Br J Cancer 102: 815-826, 2010.

30. Hardy KM, Booth BW, Hendrix MJ, Salomon DS and Strizzi L: ErbB/EGF signaling and EMT in mammary development and breast cancer. J Mammary Gland Biol Neoplasia 15: 191-199, 2010.

31. Wang KH, Kao AP, Chang CC, et al: Increasing CD $44^{+} / \mathrm{CD} 24$ tumor stem cells, and upregulation of COX-2 and HDAC6, as major functions of HER2 in breast tumorigenesis. Mol Cancer 9: $288,2010$.

32. Katoh M and Katoh M: STAT3-induced WNT5A signaling loop in embryonic stem cells, adult normal tissues, chronic persistent inflammation, rheumatoid arthritis and cancer (Review). Int J Mol Med 19: 273-278, 2007.

33. Bronowski C, Smith SL, Yokota K, et al: A subset of mucosaassociated Escherichia coli isolates from patients with colon cancer, but not Crohn's disease, share pathogenicity islands with urinary pathogenic E. coli. Microbiology 154: 571-583, 2008.

34. Bourseau-Guilmain E, Griveau A, Benoit JP and Garcion E: The importance of the stem cell marker prominin-1/CD133 in the uptake of transferrin and in iron metabolism in human colon cancer Caco-2 cells. PLoS One 6: e25515, 2011.

35. Wu WK, Sung JJ, Wu YC, et al: Inhibition of cyclooxygenase-1 lowers proliferation and induces macroautophagy in colon cancer cells. Biochem Biophys Res Commun 382: 79-84, 2009.

36. Li W, Wang J, Jiang HR, et al: Combined effects of cyclooxygenase- 1 and cyclooxygenase-2 selective inhibitors on ovarian carcinoma in vivo. Int J Mol Sci 12: 668-681, 2011.

37. Silva IA, Bai S, McLean K, et al: Aldehyde dehydrogenase in combination with CD133 defines angiogenic ovarian cancer stem cells that portend poor patient survival. Cancer Res 71: 3991-4001, 2011.

38. Kast RE and Belda-Iniesta C: Suppressing glioblastoma stem cell function by aldehyde dehydrogenase inhibition with chloramphenicol or disulfiram as a new treatment adjunct: an hypothesis. Curr Stem Cell Res Ther 4: 314-317, 2009.

39. Ghantous A, Gali-Muhtasib H, Vuorela H, Saliba NA and Darwiche N: What made sesquiterpene lactones reach cancer clinical trials? Drug Discov Today 15: 668-678, 2010.

40. Park SS, Park SK, Lim JH, Choi YH, Kim WJ and Moon SK: Esculetin inhibits cell proliferation through the Ras/ERK1/2 pathway in human colon cancer cells. Oncol Rep 25: 223-230, 2011.

41. Nakamura Y: Retracted: Modulation of p53/Akt/phosphatase and tensin homolog expression by esculetin potentiates the anticancer activity of cisplatin and prevents its nephrotoxicity. Cancer Sci 103: 154,2012

42. Furusawa S, Nakano S, Wu J, et al: Apoptosis induced by doxorubicin and cinchonine in P388 multidrug-resistant cells. J Pharm Pharmacol 53: 1029-1039, 2001.

43. Lee SY, Rhee YH, Jeong SJ, et al: Hydrocinchonine, cinchonine, and quinidine potentiate paclitaxel-induced cytotoxicity and apoptosis via multidrug resistance reversal in MES-SA/DX5 uterine sarcoma cells. Environ Toxicol 26: 424-431, 2011.

44. Donahue RN, McLaughlin PJ and Zagon IS: Low-dose naltrexone suppresses ovarian cancer and exhibits enhanced inhibition in combination with cisplatin. Exp Biol Med (Maywood) 236: 883-895, 2011.

45. Wright MH, Calcagno AM, Salcido CD, Carlson MD, Ambudkar SV and Varticovski L: Brcal breast tumors contain distinct $\mathrm{CD} 44^{+} / \mathrm{CD} 24^{-}$and $\mathrm{CD} 133^{+}$cells with cancer stem cell characteristics. Breast Cancer Res 10: R10, 2008.

46. Sauvageot CM, Weatherbee JL, Kesari S, et al: Efficacy of the HSP90 inhibitor 17-AAG in human glioma cell lines and tumorigenic glioma stem cells. Neuro Oncol 11: 109-121, 2009.

47. Tatokoro M, Koga F, Yoshida S, et al: Potential role of Hsp90 inhibitors in overcoming cisplatin resistance of bladder cancerinitiating cells. Int J Cancer 131: 987-996, 2012.

48. Yip NC, Fombon IS, Liu P, et al: Disulfiram modulated ROS-MAPK and NFкB pathways and targeted breast cancer cells with cancer stem cell-like properties. Br J Cancer 104: $1564-1574,2011$ 